\title{
Correspondence
}

\section{An extremely rare association of multiple familial trichoepitheliomas and hereditary multiple osteochondromas}

Multiple familial trichoepitheliomas (MFT) and hereditary multiple osteochondromas (HMO) are both rare and autosomal dominant disorders. Herein, we describe the first case with the two genetic syndromes together.

A 35-year-old Caucasian woman, with personal history of multiple excisions of painful osteochondromas in childhood, was observed due to a facial dermatosis that appeared 15 years ago. She indicated that her mother and maternal grandmother had multiple osteochondromas, while her paternal grandmother used to have similar facial lesions. General physical examination showed multiple palpable bone masses in the knees, arms, and forearms; short stature; ulnar shortening of the forearm; and ulnar deviation of the wrist (Bessel-Hagen deformity). Dermatologic physical examination revealed multiple, rounded, grouped, firm, and skin-colored papules scattered through the nasolabial sulcus, nose, and frontal region (Fig. 1a). No extrafacial lesions were observed. Histopathologic examination of two facial lesions demonstrated multiple nodular proliferations of uniform basaloid cells with focal peripheral palisading, numerous central keratinfilled cysts, and clefts between stromal elements or between stroma and adjacent dermis (Fig. 1b). X-ray images of the shoulders, elbows, wrists, and knees were consistent with multiple osteochondromas (Fig. 2). The genetic test found a heterozygous mutation in the exon 9 of cylindromatosis gene (CYLD) [c.1112C $>A($ p.Ser371*)], allowing the clinical diagnosis of MFT and a new heterozygous frameshift mutation never described before in the exostosin-1 gene (EXT1), leading to a change in leucine to cysteine at position 667 [c.2000del (p.Leu667Cys$\left.f s^{*} 6\right)$ ], supporting the clinical diagnosis of HMO. Since childhood she periodically visits the orthopedic department and has been submitted to multiple surgical excisions of symptomatic osteochondromas. Surgical excision and $\mathrm{CO}_{2}$ LASER has been performed in a few cutaneous lesions. The patient and the remaining family were referred to the genetic department.

Multiple familial trichoepithelioma (OMIM 601606) is characterized by multiple skin-colored small papules and nodules usually confined to the nasolabial folds, nose, forehead, and upper lip, with frequent confluence of the lesions. ${ }^{1}$ Zhang et al. ${ }^{2}$ identified for the first time mutations in the CYLD gene on chromosome 16q12-q13, responsible for MFT. The CYLD gene acts as a tumor suppressor and is critical for various signalling pathways. ${ }^{3}$ Inactivation of this gene may contribute to oncogenesis by enhancing the degradation of proteins that suppress cell proliferation or promote apoptosis. ${ }^{3,4}$ Simultaneously, it is involved in the feedback inhibition of RANK signalling pathway in the bone cells. ${ }^{5}$ Loss of $C Y L D$ results in a hyperproduction of osteoclasts, which is associated with severe bone loss or osteoporosis. ${ }^{5}$ The association with bone tumors is not clear. ${ }^{5}$

On the other hand, hereditary multiple osteochondromas (OMIM 133700) are characterized by multiple benign cartilagecapped tumors that grow outward from the metaphyses of long bones. ${ }^{6}$ Osteochondromas usually continue to grow until puberty, and clinical manifestations include short stature, bone deformities, skeletal abnormalities, compression of adjacent tissues, and impaired articular function. ${ }^{7}$ The two genes responsible for HMO, EXT1, and EXT2, are also tumor suppressors located at 8q24.11 and 11p11.2, respectively. ${ }^{6}$ They encode glycosyltransferases required for heparin sulphate (HS) biosynthesis. Heterozygous loss-of-function mutations lead to disturbed interactions and systemic HS deficiency. ${ }^{8} \mathrm{HS}$ is involved in the regulation signalling of endochondral ossification and, thereby, relevant to the normal skeletal development. ${ }^{9}$ Additionally, HS also plays a vital role in hair follicle morphogenesis.
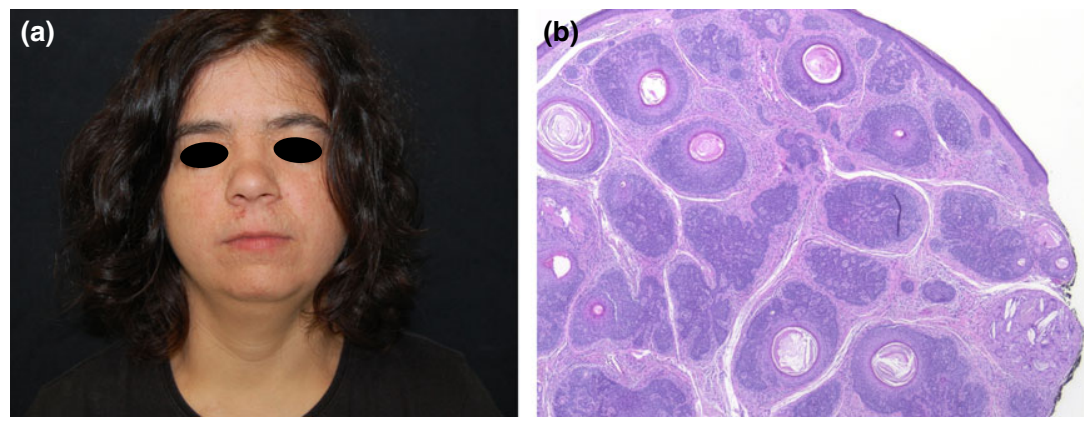

Figure 1 (a) Multiple skin-colored papules and nodules on the face; (b) histological examination of a facial lesion showed multiple nodular proliferations of uniform basaloid cells with focal peripheral palisading and small keratin-filled cysts compatible with trichoepithelioma $(\mathrm{H} \& \mathrm{E}, \times 40)$ 

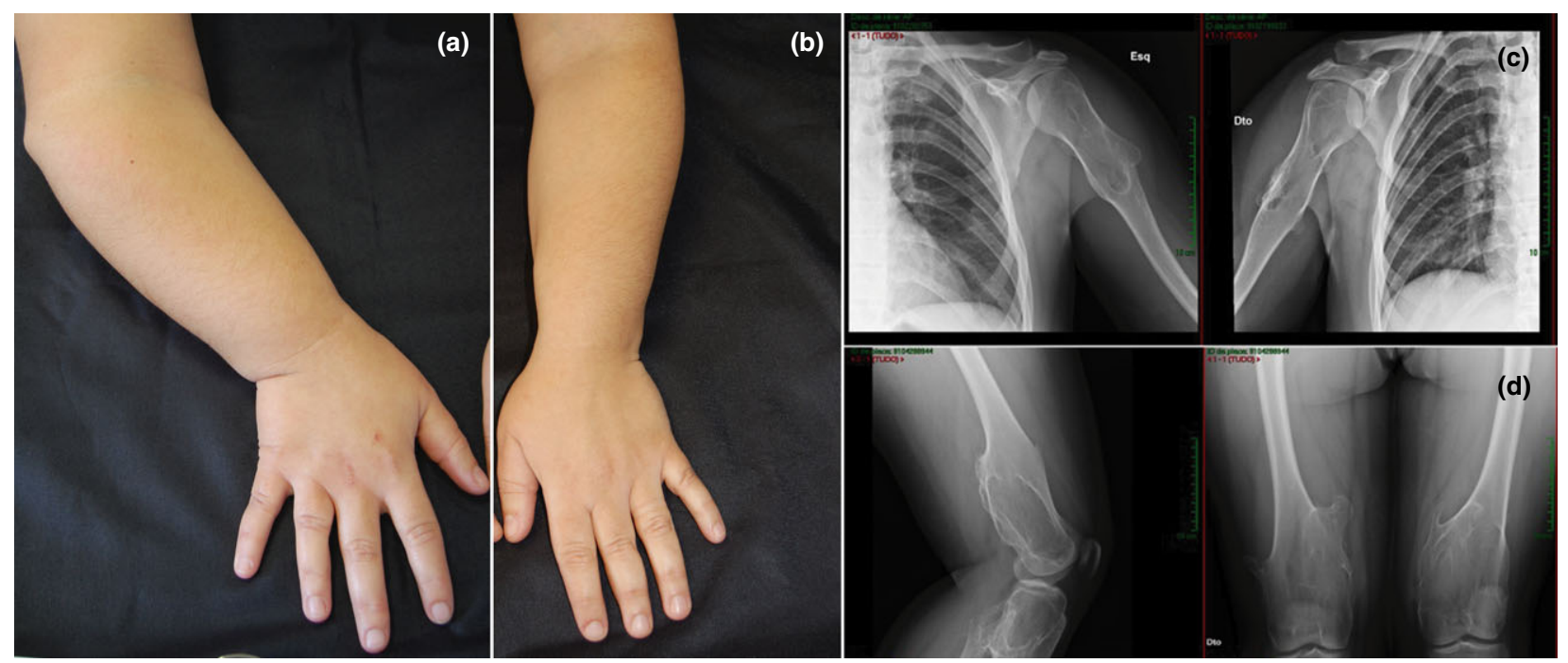

Figure 2 (a) Ulnar shortening of the forearm and ulnar deviation of the wrist (Bessel-Hagen deformity); (b) Shoulders, elbows, and wrists x-ray images showed multiple osteochondromas

The mutation in EXT gene can lead to a dysregulated hair follicle morphogenesis and consequently to the development of benign adnexal neoplasm as trichoepitheliomas. ${ }^{10}$

Here we reported for the first time a coexistence of multiple trichoepitheliomas and multiple osteochondromas in a carrier of two germline mutations in CYLD and EXT1 genes. The genetic analysis allowed us to diagnose MFT and HMO, two well-known hereditary syndromes never described before together in a single patient and with a new heterozygous frameshift mutation in the EXT1 gene.

\section{Ana F. Monteiro ${ }^{1 *}$, MD (iD Francisco Saraiva Gil ${ }^{1}$, MD Margarida Rato ${ }^{1}$, MD Tomás Osório ${ }^{2}$, MD Ermelindo Tavares $^{3}$, MD}

${ }^{1}$ Dermatovenereology Department, Hospital de Santarém EPE, Santarém, Portugal

${ }^{2}$ Department of Orthopedic Surgery, Unidade Local de Saúde do Baixo Alentejo, EPE - Hospital José Joaquim Fernandes,

Beja, Portugal

${ }^{3}$ Dermatovenereology Department, Hospital de Vila Franca de Xira, Vila Franca de Xira, Portugal *E-mail: anafilipemonteiro@gmail.com

Funding: None.

Conflicts of interest: None.

doi: $10.1111 / \mathrm{ijd} .14251$

\section{References}

1 Kazakov DV. Brooke-Spiegler syndrome and phenotypic variants: an update. Head Neck Pathol 2016; 10: 125-130.

2 Zhang X-J, Liang Y-H, He P-P, et al. Identification of the cylindromatosis tumor-suppressor gene responsible for multiple familial trichoepithelioma. J Invest Dermatol 2004; 122: 658664.

3 Ying Z-X, Ma H-Q, Liu Y, et al. A novel mutation of CYLD in a Chinese family with multiple familial trichoepithelioma. J Eur Acad Dermatol Venereol 2012; 26: 1420-1423.

4 Brummelkamp TR, Nijman SMB, Dirac AMG, et al. Loss of the cylindromatosis tumour suppressor inhibits apoptosis by activating NF- $\kappa$ B. Nature 2003; 424: 797-801.

5 Sun S-C. CYLD: a tumor suppressor deubiquitinase regulating NF-kappaB activation and diverse biological processes. Cell Death Differ 2010; 17: 25-34.

$6 \mathrm{Li} \mathrm{Y,} \mathrm{Wang} \mathrm{J,} \mathrm{Wang} \mathrm{Z,} \mathrm{et} \mathrm{al.} \mathrm{A} \mathrm{genotype-phenotype} \mathrm{study} \mathrm{of}$ hereditary multiple exostoses in forty-six Chinese patients. BMC Med Genet 2017; 18: 126.

7 Guo X, Deng Y, Liu H. Clinical characteristics of hereditary multiple exostoses: a retrospective study of mainland chinese cases in recent 23 years. $J$ Huazhong Univ Sci Technolog Med Sci 2014; 34: 42-50.

8 Pacifici M. The pathogenic roles of heparan sulfate deficiency in hereditary multiple exostoses. Matrix Biol 2017; in press. https:// doi.org/10.1016/j.matbio.2017.12.011

9 Matsumoto $\mathrm{Y}$, Matsumoto K, Irie F, et al. Conditional ablation of the heparan sulfate-synthesizing enzyme Ext1 leads to dysregulation of bone morphogenic protein signaling and severe skeletal defects. J Biol Chem 2010; 285: 19227-19234.

10 Coulson-Thomas VJ, Gesteira TF, Esko J, et al. Heparan sulfate regulates hair follicle and sebaceous gland morphogenesis and homeostasis. J Biol Chem 2014; 289: 25211-25226. 